\title{
Constitutively active Stat5A and Stat5B promote adipogenesis
}

\author{
Hiroshi Wakao • Rika Wakao • Atsushi Oda • \\ Hiroyoshi Fujita
}

Received: 13 September 2010/Accepted: 1 November 2010/Published online: 1 December 2010

(C) The Japanese Society for Hygiene 2010

\begin{abstract}
Objective The metabolic syndrome is an important social problem affecting many people in developed countries. Obesity is a leading cause of this syndrome, hence understanding molecular mechanisms underlying obesity is of prime importance for preventive medicine to develop novel methods to alleviate the corresponding social cost as well as for pharmaceutical companies to develop antimetabolic drugs.

Methods Since adipocytes play an important role in obesity, we explored the signaling pathways leading to differentiation of adipocytes. We used a preadipocyte cell line to monitor the differentiation of adipocytes, and virusmediated gene transfer to assess the role of the transcription factor Stat5 in adipogenesis. Adipocyte differentiation was assessed by Northern blot and Western blot analyses as well as accumulation of fat droplets in cells. Promoter activity of the proadipogenic transcription factor peroxisome proliferator-activated receptor-gamma (PPAR $\gamma)$ was evaluated by luciferase assay.

Results Virus-mediated gene transfer of the constitutively active form of both Stat5A and Stat5B resulted in enhanced adipocyte differentiation in the absence of fetal bovine serum (FBS) as judged by expression of proadipogenic factors as well as accumulation of fat droplets in cells.
\end{abstract}

H. Wakao $(\bowtie) \cdot$ A. Oda $\cdot$ H. Fujita

Department of Environmental Biology,

School of Medicine, Hokkaido University,

North 15 West 7, Kita-ku, Sapporo 060-8638, Japan

e-mail: hwakao@med.hokudai.ac.jp

R. Wakao

Pharmaceutical and Medical Devices Agency,

3-3-2 Kasumigaseki, Chiyoda-ku,

Tokyo 100-0013, Japan
Such a proadipogenic effect of Stat5 is, in part, mediated by its ability to enhance transcription of PPAR $\gamma$, a master transcriptional regulator in adipogenesis.

Conclusion The constitutively active form of Stat5A and Stat5B promoted adipocyte differentiation in the absence of FBS via induction of PPAR $\gamma$.

Keywords Obesity $\cdot$ Adipocytes - Stat5 .

Signal transduction $\cdot \operatorname{PPAR} \gamma$

\section{Introduction}

At present, more than 1.7 billion people are considered to be obese [1]. Obesity is caused by excess calorie intake and is a major worldwide health concern. The World Health Organization reports that at least 170 million people worldwide suffer from diabetes due to obesity. Obesity is characterized by dysregulated metabolism, dyslipidemia, insulin resistance, metabolic syndrome, nonalcoholic fatty liver disease, hyperglycemia, hypertension, some forms of cancer, and increased risk for development of type 2 diabetes (T2D) and its most serious comorbidity, cardiovascular disease. It is estimated that around $90 \%$ of T2D is due to obesity [2]. If not reversed, exacerbating obesity will lead to an epidemic of these comorbidities that will challenge many health care delivery systems. Thus, obesity poses a great challenge for public health.

To date, many studies have shed light on obesogenic genes, transcriptional processes, and signaling pathways that control weight gain; however, we are far from understanding the whole process of obesity [3, 4]. Since excess adipocyte size and number are tightly linked to obesity, it is important to decipher the molecular mechanisms underlying such differentiation. 
Differentiation of preadipocyte cell lines such as 3T3L-1 and $3 \mathrm{~T} 3-\mathrm{F} 422 \mathrm{~A}$ is stimulated in response to adipogenic hormone cocktails comprising insulin, glucocorticoids, cyclic adenosine monophosphate (cAMP)-producing factors, and fetal bovine serum (FBS). Such treatment allows growth-arrested preadipocytes to re-enter the cell cycle with another one or two cell cycles, which is known as mitotic clonal expansion. The transcription factor CCAAT/ enhancer binding proteins (C/EBP) $\beta$ and $\delta$ are induced during this period, and induction of these factors is a prerequisite for expression of $\operatorname{PPAR} \gamma$, a master regulator of adipogenesis [3, 4]. PPAR $\gamma$ in turn stimulates expression of $\mathrm{C} / \mathrm{EBP} \alpha \cdot \operatorname{PPAR} \gamma$ and $\mathrm{C} / \mathrm{EBP} \alpha$ promote adipogenesis by turning on adipocyte-specific genes such as aP2, leptin, and adiponectin. Terminally differentiated adipocytes harbor fat droplets within the cells, which is a hallmark of energystoring cells.

$\operatorname{PPAR} \gamma$ is a nuclear hormone receptor whose agonists such as thiazolidinedione (TZD) troglitazone, and rosiglitazone are used as antidiabetic drugs, though the natural ligand in the body has yet to be determined. It is noteworthy that PPAR $\gamma$ ligands are required for efficient adipogenesis of fibroblastic cells [5].

The role of glucocorticoids and cAMP-producing factors in adipogenesis is considered to be induction of $\mathrm{C} / \mathrm{EBP} \delta$ and $\beta$, respectively, while that of insulin is believed to be cross-activation of insulin-like growth factor (IGF)-1 receptor. In contrast, the role of FBS in adipogenesis is more ambiguous, although FBS is essential for adipogenesis. It is proposed that IGF-1 in FBS is critical for adipogenesis; however, this remains to be formally proven.

Stat family proteins Stat1, 2, 3, 4, 5A, 5B, and 6 are activated by many cytokines and play crucial roles in cell proliferation and differentiation [6]. That the size of the fat pad from Stat5A and Stat5B double-knockout mice is onefifth of that from control mice suggests that Stat5 plays a role in adipogenesis [7]. Concurrently we have shown that Stat5 is activated by FBS and adipogenic hormones, and conveys the extracellular stimulus to the nucleus, culminating in execution of the adipogenic program in 3T3-L1 cells. Activation of Stat5 tightly correlates with expression of PPAR $\gamma 2$, an isoform of PPAR $\gamma$ exclusively expressed in adipocytes [8].

In the present report, we show that Stat5 regulates murine PPAR $\gamma 2$ promoter activity. Furthermore, we demonstrate that ectopic expression of the constitutively active form of Stat5A or Stat5B promotes expression of PPAR $\gamma 2$ and adipogenesis in the absence of FBS. These data reveal a novel facet of transcriptional cascades in adipogenesis and highlight the role of Stat5 in fat cell differentiation.

\section{Materials and methods}

Retrovirus-mediated gene transduction in 3T3-L1 cells

Retroviral vectors were constructed using pLNCX2 (Clontech). pLNCX2 harboring the constitutively active form of murine Stat5A (Stat5A*) and Stat5B (Stat5B*) [9] were generated using the Quick change mutation kit (Stratagene), and the mutated DNA sequence was verified (ABI, 310 sequencer). These Stat5s reside in the nucleus and show constitutive transcriptional activity in the absence of ligands. Recombinant retroviruses were produced using Ecopack2 packaging cells and used for transduction (Clontech). Neomycin-resistant 3T3-L1 cells were selected after 2 weeks. These cells were grown to confluence and subjected to adipogenic regimen.

Cell culture and induction of differentiation

3T3-L1 cells were maintained in growth medium consisting of Dulbecco's modified Eagle's medium (DMEM) containing $10 \%$ normal calf serum (CS). To differentiate 3T3-L1 cells, they were grown to confluence and left for 2 days. Cells were then exposed to fresh differentiation medium consisting of DMEM, $10 \% \mathrm{CS}, 1 \mu \mathrm{M}$ dexamethasone (DEX), $0.5 \mathrm{mM}$ methylisobutylxanthine (MIX), and $10 \mu \mathrm{g} / \mathrm{ml}$ insulin for $48 \mathrm{~h}$. Thereafter, cells were refed every other day with DMEM containing 10\% CS and $2.5 \mu \mathrm{g} / \mathrm{ml}$ insulin. All experiments were done with CS, except the transcriptional assay as shown in Fig. 1b, c, wherein FBS was used instead of CS. FBS contains some factors that activate Stat5.

\section{Transient transfection assay}

Murine PPAR $\gamma 2$ promoter comprising +1 to -1047 or +1 to -1029 (devoid of Stat5 binding site) was cloned from the genomic DNA of C57BL/6 mice by polymerase chain reaction (PCR) with Pfu-turbo DNA polymerase (Stratagene) and ligated to pGL2-basic luciferase reporter gene (Clontech). PPAR $\gamma 2$ promoter harboring a mutated Stat5 site $\left(5^{\prime}\right.$-TTCAGGGAA- $3^{\prime}$ to $5^{\prime}$-GGCAGGTAA- $\left.3^{\prime}\right)$ was created by PCR. Transfection of reporter gene was performed with Lipofectamine-plus (Invitrogen) together with Stat5A, Stat5B, Stat $5 A^{*}$ or Stat5B* complementary DNA (cDNA) in the presence of cDNA for renilla luciferase as internal control. After transfection, both firefly and renilla luciferase activities were measured, and relative luciferase activity was calculated. 

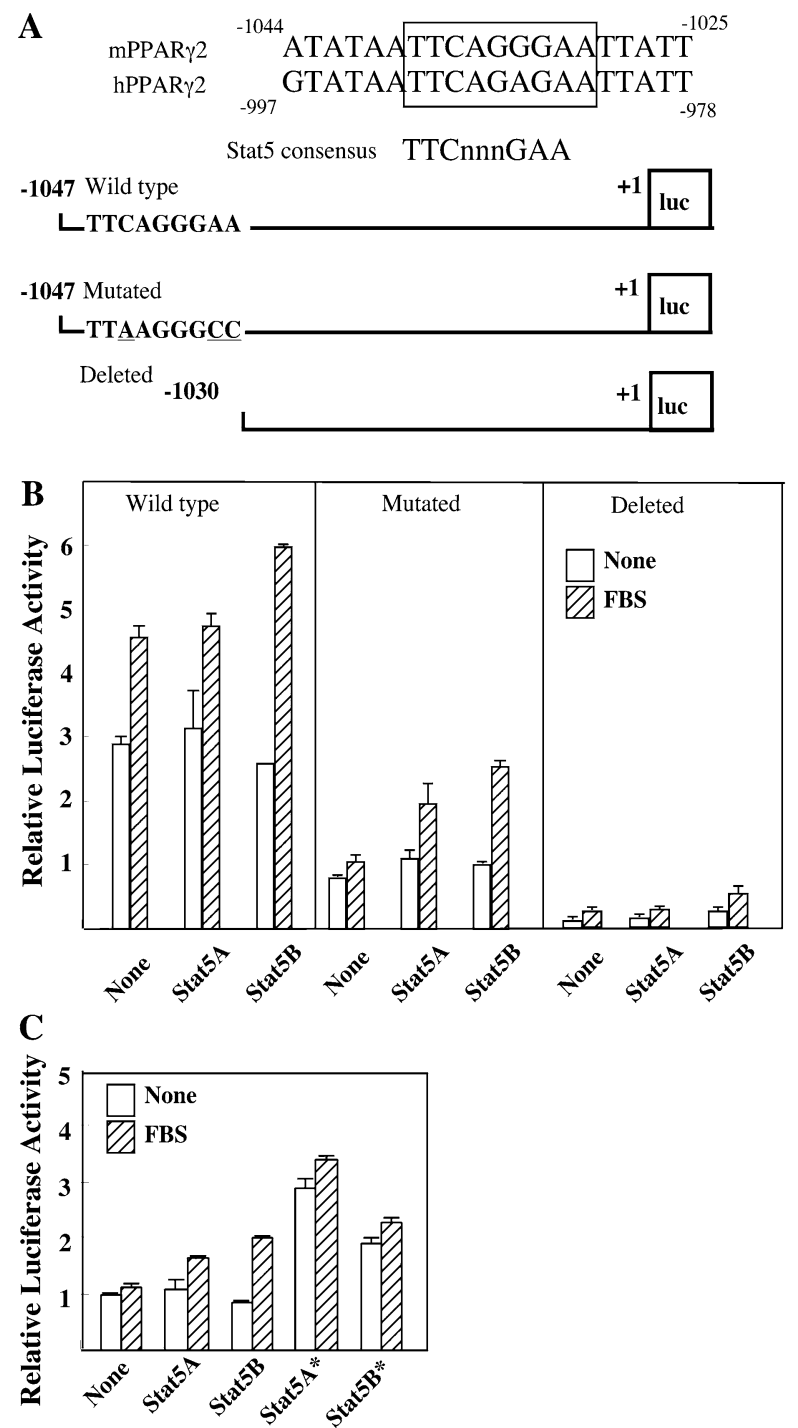

Fig. 1 Stat5 activates the PPAR $\gamma 2$ promoter. a The sequence of murine and human PPAR $\gamma 2$ gene promoter was analyzed, and a potential Stat 5 binding site in the promoters is indicated by the closed box (upper panel). Also is indicated a consensus Stat5 binding site. Schematic representation of the reporter gene constructs containing a wild-type Stat5 binding site (wild type), mutated Stat5 binding site (mutated nucleotides are underlined) or no Stat5 binding site (deleted) (lower panel). b Stat5 transactivates PPAR $\gamma 2$ promoter via Stat5responsive element. Relative luciferase activity (firefly luciferase activity/renilla luciferase activity) was determined with the reporter constructs containing a Stat 5 binding site, a mutated Stat 5 binding site or no Stat5 binding site. After serum starvation, cells were left unstimulated or stimulated with FBS for $6 \mathrm{~h}$. Results are expressed as fold induction and represent the mean \pm standard deviation of three independent experiments. c Constitutively active Stat5A and Stat5B activate the PPAR $\gamma 2$ promoter in an FBS-independent manner. 3T3L1 cells were transfected with the PPAR $\gamma 2$ reporter construct containing a Stat5 binding site together with wild-type or constitutively active Stat5 complementary DNA (cDNA) (Stat5A* or Stat5B*). After starvation, cells were left unstimulated or stimulated with $10 \%$ FBS for $6 \mathrm{~h}$. Results are from three independent experiments performed in triplicates
RNA preparation

Total RNA was prepared with Trizol solution (Invitrogen) at the indicated time points and subjected to Northern blot analysis with digoxigenin (DIG)-labeled antisense RNA probes as described [8].

Western blot analysis

Total cell lysates from 3T3-L1 cells were prepared 10 days after differentiation. Proteins $(50 \mu \mathrm{g})$ were separated on a sodium dodecyl sulfate (SDS) polyacrylamide gel and transferred to polyvinylidene fluoride (PVDF) membrane. Proteins were visualized by enhanced chemiluminescence (ECL) system (GE Biosciences). Anti-Stat5 (C-17), anti$\operatorname{PPAR} \gamma(\mathrm{H}-100)$, and anti-C/EBP $\alpha$ (14AA) antibodies were purchased (Santa Cruz Biotechnology).

\section{Results}

We have previously shown that Stat5 overexpression promotes PPAR $\gamma 2$ expression, while ectopic expression of dominant negative Stat 5 compromises it at both messenger RNA (mRNA) and protein levels [8]. To gain insight into the molecular mechanism underlying PPAR $\gamma 2$ mRNA expression, we have aligned a putative promoter region of murine and human PPAR $\gamma 2$ that is specific for adipocytes [10]. Sequence comparison revealed that they exhibited high similarity; in particular, several C/EBP binding sites known to be important for PPAR $\gamma 2$ promoter activity were well conserved [11]. Intriguingly, sequences of mouse promoter comprising between -1038 and -1030 and the corresponding human region contained a putative Stat5 binding site (TTCNNNGAA, N: any nucleotide) [12] (Fig. 1a, upper panel). These data indicate that the Stat5 binding site might be important for PPAR $\gamma 2$ promoter activity.

We explored this by measuring the murine PPAR $\gamma 2$ promoter-luciferase reporter activity. Reporter gene construct containing a putative Stat5 binding site was transfected into 3T3-L1 cells together with Stat5A or Stat5B cDNA. After transfection, cells were left unstimulated or stimulated with FBS, and relative luciferase activity was measured. In the absence of FBS, luciferase activity was barely affected by exogenous Stat5A or Stat5B, but a certain level of basal activity was observed. This was most likely due to endogenous Stat5s or other Stat family proteins (Fig. 1b, none). While stimulation with FBS augmented luciferase activity, ectopic expression of Stat5A or Stat5B further increased luciferase activity by $2.5-$ or 
3-fold, respectively (Fig. 1b, wild type, FBS). In sharp contrast, mutation in the putative Stat5 binding site severely compromised activity in response to FBS (Fig. 1b, mutated). Furthermore, ablation of the putative Stat5 binding site virtually abolished promoter activity, regardless of stimulation (Fig. 1b, deleted). These results indicate that Stat5 controls, at least partially, the activity of the murine PPAR $\gamma 2$ promoter, and is responsible for mediating FBS-induced expression of PPAR $\gamma 2$.

Several reports have shown that FBS is an essential factor for adipogenesis, although its effector molecules in FBS have not yet been identified. Our previous data suggest that FBS plays a role in inducing PPAR $\gamma 2$ in 3T3-L1 as well as NIH3T3 cells [13]. Since FBS enhanced PPAR $\gamma 2$ promoter activity via Stat5 binding site (Fig. 1b), we hypothesized that constitutively active Stat5s might obviate the FBS dependence of 3T3-L1 cells in adipocyte differentiation. To explore this, we evaluated the effect of constitutively active Stat5 (hereafter designated Stat5A* or Stat5B*) on PPAR $\gamma 2$ promoter activity. Intriguingly, introduction of Stat5A* and to a lesser extent Stat5B* resulted in further enhanced activity. In both cases, however, there was little difference in transcriptional activity regardless of stimulation by FBS (Fig. 1c). These results indicate that constitutively active Stat5 substitutes the function of FBS for inducing PPAR $\gamma 2$ in 3T3-L1 cells.

We then examined whether ectopic expression of Stat5A* or Stat5B* culminated in promotion of $3 \mathrm{~T} 3-\mathrm{L} 1$ cell adipogenesis in the absence of FBS. To this end, Stat5A* or Stat5B* was introduced into 3T3-L1 cells by retrovirus. After transduction, cells were induced to differentiate in the presence of MIX, DEX, and insulin with CS. As a control, cells harboring the empty vector were subjected to an identical regimen. The effects of constitutively active Stat5 were assessed by Northern blot analysis for transcription factors relevant to adipogenesis, such as $C / E B P \beta, \delta$, and $\alpha, P P A R \gamma$, and SREBPIc (Fig. 2a). Both Stat5A* and Stat5B* augmented C/EBP $\beta$ mRNA, while they had little impact on $\mathrm{C} / \mathrm{EBP} \delta$ mRNA during adipogenesis (Fig. 2a). As for C/EBP $\alpha$ mRNA, neither Stat $5 A^{*}$ nor Stat5B* showed any significant effect until day 4. However, both Stat5s maintained the high level of the transcript up to day 10 , whereas the empty vector failed to do so. Stat5A* and Stat5B* led to an increment of SREBP1c mRNA over the control, whilst the former exhibited slightly stronger effects than the latter. The impact of constitutively active Stat5 expression was most pronounced in PPAR $\gamma 2$ mRNA. Whereas the empty vector alone barely induced PPAR $\gamma 2$ mRNA, ectopic expression of Stat5A* and Stat5B* markedly stimulated it from day 4 to 10 without any decline (Fig. 2a). Together, these results demonstrated that constitutively active Stat5A and Stat5B enhanced mRNA expression for proadipogenic factors such
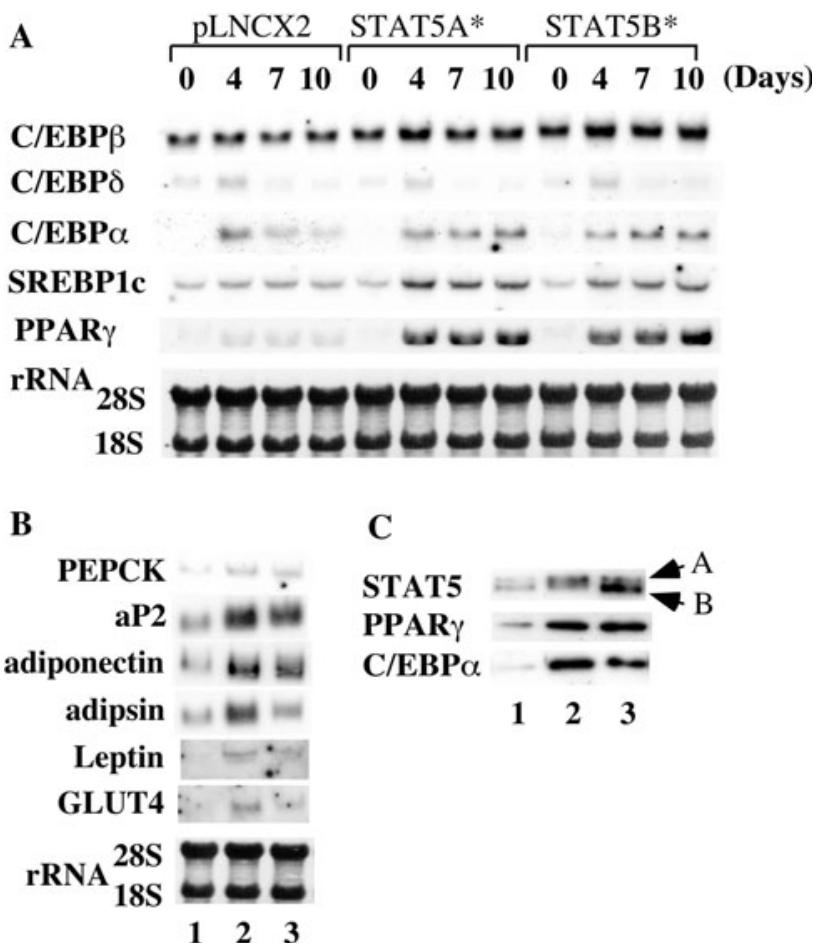

Fig. 2 Constitutively active Stat5A and Stat5B promote expression of proadipogenic factors. a Ectopic expression of constitutively active Stat5s induces proadipogenic transcription factors. 3T3-L1 cells were infected with control retrovirus vector ( $\mathrm{pLNCX} 2)$, vector harboring constitutively active Stat5A (Stat5A*) or Stat5B (Stat5B*). After G418 selection, cells were grown to confluence and subjected to the adipogenic regimen as described in the "Materials and methods" section. Total RNA was extracted at the indicated time, and $2 \mu \mathrm{g}$ of each sample was subjected to Northern blot analysis with the antisense riboprobes corresponding to the genes. Three independent experiments were performed, and essentially identical results were obtained. b Induction of adipogenic marker genes in cells transduced with constitutively active Stat5s. Total RNA was isolated at 10 days post differentiation induction and subjected to Northern blot analysis with DIG-labeled riboprobes. Lane 1 cells transduced with the empty vector, lane 2 cells harboring Stat5A*, lane 3 cells harboring Stat5B*. c Increase of $\mathrm{C} / \mathrm{EBP} \alpha$ and $\mathrm{PPAR} \gamma$ proteins upon Stat5 transduction. Cells harboring empty vector, Stat $5 A^{*}$ or Stat $5 B *$ were subjected to the adipogenic regimen. Ten days later, cells were harvested and cell lysates were prepared. After separation on SDS gel, the proteins were visualized by the indicated antibodies using an ECL system. For Stat5, position of Stat5A and Stat5B is depicted by arrow. Lane 1 cells transduced with the empty vector, lanes 2 and 3 cells harboring Stat $5 \mathrm{~A}^{*}$ or Stat5B*, respectively

as $\mathrm{C} / \mathrm{EBP} \beta$, SREBP1c, and PPAR $\gamma 2$ in the absence of FBS in 3T3-L1 cells.

We then investigated whether such an increase in proadipogenic factors led to adipogenesis by biochemical and morphological criteria. Several terminal markers for adipocytes were assessed by Northern blot analysis at the end of differentiation (Fig. 2b). Phosphoenolpyruvate carboxykinase (PEPCK) mRNA was slightly but significantly elevated in cells harboring Stat5A* or Stat5B* over the control (Fig. 2b). Stat5A* more strongly induced mRNA 
for aP2, adiponectin, adipsin, leptin, and glucose transporter 4 (Glut4) than did Stat5B* (Fig. 2b, lanes 1-3). It is worth noting that preferential activation of adipsin and Glut4 was also observed in NIH3T3 cells harboring Stat5A [14]. Nevertheless, such a biased increment of the transcript for aP2 and PPAR $\gamma 2$ in NIH3T3 cells was not evident in 3T3-L1 cells harboring Stat5A* (Fig. 2a, b).

Since PPAR $\gamma 2$ and $\mathrm{C} / \mathrm{EBP} \alpha$ are critical factors for adipogenesis, the correlation between ectopic expression of Stat5 and resulting induction of such factors was evaluated by Western blot analysis (Fig. 2c). Ectopic expression of Stat5A* or Stat5B* proteins was evident (Fig. 2c, lanes 1-3). While empty vector alone led to a low level expression of PPAR $\gamma 2$ and $\mathrm{C} / \mathrm{EBP} \alpha$, ectopic expression of Stat $5 \mathrm{~A} *$ or Stat $5 \mathrm{~B} *$ significantly increased these proteins. Stat5A* was more potent than Stat5B* for inducing $\mathrm{C} / \mathrm{EBP} \alpha$ (Fig. 2c, lanes 1-3). These results mirrored the strong activity of Stat5A* in inducing mRNA for aP2, adiponectin, adipsin, leptin, and Glut4.

Finally, adipogenesis was assessed by Oil-Red-O staining, which detects neutral lipids. While little staining was observed in cells transduced with the empty vector, cells harboring Stat5A* or Stat5B* showed substantial staining and rounded-up morphology (Fig. 3, compare 1-3). These results were in accordance with the simultaneous augmentation of genes relevant to mature adipocytes such as $P P A R \gamma$, adiponectin, aP2, and PEPCK (Fig. 2a, b).

\section{Discussion}

Adipogenic hormones MIX, a cAMP inducer, and DEX are responsible for transcriptional induction of $\mathrm{C} / \mathrm{EBP} \beta$ and
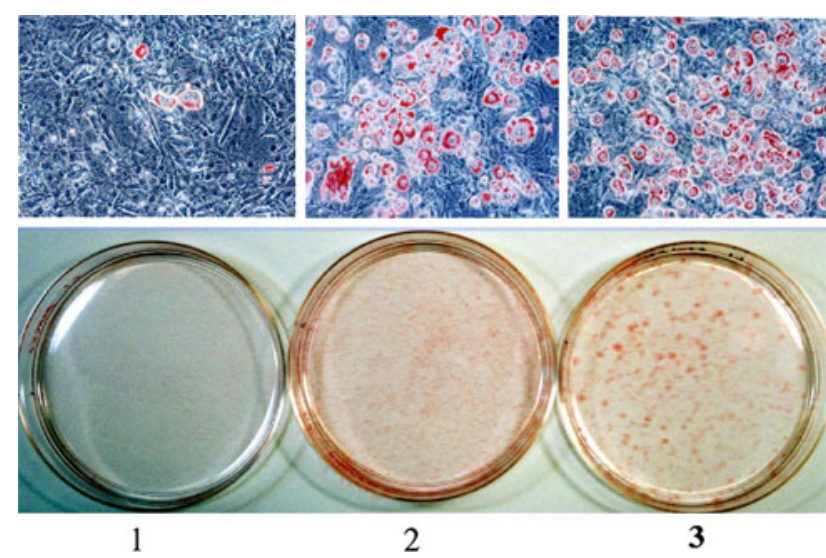

Fig. 3 Impact of constitutively active Stat5 on triglyceride loading (Oil-Red-O staining of neutral lipids). 3T3-L1 cells were differentiated as described in Fig. 2. After 10 days, cells were stained with OilRed-O to visualize triglyceride vesicles. Upper panel microscopic view, lower panel macroscopic view. Lane 1 cells transduced with the empty vector, lanes 2 and 3 cells harboring Stat5A* or Stat5B*, respectively
$\mathrm{C} / \mathrm{EBP} \delta$, respectively, at the early phase of adipocyte differentiation [15]. Ectopic expression of these factors leads to adipogenic conversion of NIH3T3 or 3T3-L1 cells by inducing $\operatorname{PPAR} \gamma$, which then results in execution of adipogenesis [16, 17]. Conversely, ablation of these factors severely compromised adipocyte differentiation [3]. Furthermore, $\mathrm{C} / \mathrm{EBP} \beta$ and $\delta$ expression is a prerequisite for $\mathrm{C} / \mathrm{EBP} \alpha$ induction [17]. Intriguingly, $\operatorname{PPAR} \gamma$ turns on expression of $\mathrm{C} / \mathrm{EBP} \alpha \cdot \mathrm{C} / \mathrm{EBP} \alpha$ itself can bind to the $\mathrm{C} / \mathrm{EBP}$ site in the $\operatorname{PPAR} \gamma$ promoter, providing a stable, positive forward regulatory loop [5]. In addition to the above classical C/EBP-PPAR $\gamma$ axis, several lines of evidence have suggested that Stat5 may play an important role in adipogenesis. Indeed, Stat5 is rapidly activated upon adipogenic hormone challenge in 3T3-L1 preadipocytes. Furthermore, overexpression of Stat5 results in promotion of adipogenesis, whilst expression of dominant negative Stat5 leads to inhibition of adipocyte differentiation. Intriguingly, the level of Stat5A and Stat5B protein increases as cells differentiate into adipocytes [8, 18]. Furthermore, FBS is a prerequisite for efficient induction of PPAR $\gamma$ mRNA in NIH3T3 as well as 3T3-L1 cells [13]. Thus, it is most likely that the requirement for FBS for efficient adipogenesis correlates with the activity of Stat5. In line with this hypothesis, prolactin and growth hormone, both of which activate Stat5, are shown to promote adipogenesis in the absence/presence of FBS in 3T3-L1 and 3T3-F422A cells, respectively [19-21]. In this regard, the weak induction of $\operatorname{PPAR} \gamma$ and $\mathrm{C} / \mathrm{EBP} \alpha$ in adipogenesis at both protein and mRNA levels most likely reflects the fact that CS fails to activate Stat5s strongly with the control vector (Fig. 2a, c). Although prolactin and growth hormone are components of FBS, it is difficult to evaluate the relative contribution of these hormones to adipogenesis, as identification of the factor(s) responsible for Stat5 activation in FBS has yet to be achieved. Intriguingly, it is reported that only Stat5A but not Stat5B can confer the adipogenic potential to the cells [14]. In this regard, our present data indicating that constitutively active Stat5A as well as Stat5B enhance expression of PPAR $\gamma 2$, and to a lesser extent $\mathrm{C} / \mathrm{EBP} \beta$, and promote adipogenesis without affecting $\mathrm{C} / \mathrm{EBP} \delta$ expression shed light on a novel adipogenic transcriptional cascade. Though both Stat5A and Stat5B appear to control PPAR $\gamma 2$ expression by interacting with its promoter, it is yet to be examined how these Stat5s impinge upon $\mathrm{C} / \mathrm{EBP} \beta$ expression.

The difference between Stat5A and Stat5B in terms of adipogenesis-enhancing potential indicates that they function differently in fat cell development (Fig. 2b). Our observation that Stat5A is more potent in inducing adipocyte genes such as PEPCK, aP2, adiponectin, adipsin, leptin, and GLUT4 is in accordance with the previous report that Stat5A can promote adipogenesis [14]. Since 
both Stat5A and Stat5B possess similar potential in inducing PPAR $\gamma 2$ and adipocyte differentiation as judged by Western blot analysis and Oil-Red-O staining (Figs. 2c, 3 ), the target genes for each Stat5, which may be responsible for optimal adipogenesis, are yet to be delineated. Identification of such genes will shed light on the molecular mechanism underlying the different adipogenic potential between Stat5A and Stat5B and opens a novel avenue for increasing our understanding of adipogenesis, which ultimately may culminate in the development of antiobesity drugs.

\section{Conclusions}

Both constitutively active Stat5A and Stat5B could functionally bypass the FBS requirement for adipogenesis in 3T3-L1 cells. These Stat5s induced PPAR $\gamma$, a master regulator in adipogenesis, thus culminating execution of the adipogenic program. Our present data suggest that compounds that stimulate the Stat5-PPAR $\gamma$ axis in adipocytes may be beneficial for promoting adipogenesis, which in turn increases fat storage in the body and alleviates metabolic syndrome [22]. Thus, the search for such compounds may open the door for future preventive medicines and contribute to improved social welfare.

Acknowledgments The authors thank Helix Institute for supporting the work.

Conflict of interest No conflict of interest.

\section{References}

1. Haslam DW, James WP. Obesity. Lancet. 2005;366:1197-209.

2. Hossain P, Kawar B, El Nahas M. Obesity and diabetes in the developing world—a growing challenge. N Engl J Med. 2007; 356:213-5.

3. Rosen ED, Spiegelman BM. Molecular regulation of adipogenesis. Annu Rev Cell Dev Biol. 2000;16:145-71.

4. Rosen ED, Spiegelman BM. Adipocytes as regulators of energy balance and glucose homeostasis. Nature. 2006;444:847-53.

5. Tontonoz P, Spiegelman BM. Fat and beyond: the diverse biology of PPARgamma. Annu Rev Biochem. 2008;77:289-312.

6. Darnell JE Jr. STATs and gene regulation. Science. 1997;277: 1630-5.

7. Teglund S, McKay C, Schuetz E, van Deursen JM, Stravopodis D, Wang D, et al. Stat5a and Stat5b proteins have essential and nonessential, or redundant, roles in cytokine responses. Cell. 1998;93:841-50.
8. Nanbu-Wakao R, Morikawa Y, Matsumura I, Masuho Y, Muramatsu MA, Senba E, et al. Stimulation of 3T3-L1 adipogenesis by signal transducer and activator of transcription 5. Mol Endocrinol. 2002;16:1565-76.

9. Onishi M, Nosaka T, Misawa K, Mui AL, Gorman D, McMahon $\mathrm{M}$, et al. Identification and characterization of a constitutively active STAT5 mutant that promotes cell proliferation. Mol Cell Biol. 1998;18:3871-9.

10. Tontonoz P, Hu E, Graves RA, Budavari AI, Spiegelman BM. mPPAR gamma 2: tissue-specific regulator of an adipocyte enhancer. Genes Dev. 1994;8:1224-34.

11. Shi XM, Blair HC, Yang X, McDonald JM, Cao X. Tandem repeat of C/EBP binding sites mediates PPARgamma2 gene transcription in glucocorticoid-induced adipocyte differentiation. J Cell Biochem. 2000;76:518-27.

12. Gouilleux F, Wakao H, Mundt M, Groner B. Prolactin induces phosphorylation of Tyr694 of Stat5 (MGF), a prerequisite for DNA binding and induction of transcription. EMBO J. 1994;13: 4361-9.

13. Nanbu-Wakao R, Fujitani Y, Masuho Y, Muramatu M, Wakao H. Prolactin enhances CCAAT enhancer-binding protein-beta $(\mathrm{C} /$ EBP beta) and peroxisome proliferator-activated receptor gamma (PPAR gamma) messenger RNA expression and stimulates adipogenic conversion of NIH-3T3 cells. Mol Endocrinol. 2000;14: 307-16.

14. Floyd ZE, Stephens JM. STAT5A promotes adipogenesis in nonprecursor cells and associates with the glucocorticoid receptor during adipocyte differentiation. Diabetes. 2003;52:308-14.

15. Cao Z, Umek RM, McKnight SL. Regulated expression of three C/EBP isoforms during adipose conversion of 3T3-L1 cells. Genes Dev. 1991;5:1538-52.

16. Wu Z, Xie Y, Bucher NL, Farmer SR. Conditional ectopic expression of C/EBP beta in NIH-3T3 cells induces PPAR gamma and stimulates adipogenesis. Genes Dev. 1995;9:2 350-63.

17. Yeh WC, Cao Z, Classon M, McKnight SL. Cascade regulation of terminal adipocyte differentiation by three members of the C/EBP family of leucine zipper proteins. Genes Dev. 1995;9: $168-81$.

18. Stephens JM, Morrison RF, Pilch PF. The expression and regulation of STATs during 3T3-L1 adipocyte differentiation. J Biol Chem. 1996;271:10441-4.

19. Shang CA, Waters MJ. Constitutively active signal transducer and activator of transcription 5 can replace the requirement for growth hormone in adipogenesis of 3T3-F442A preadipocytes. Mol Endocrinol. 2003;17:2494-508.

20. Stewart WC, Baugh JE Jr, Floyd ZE, Stephens JM. STAT 5 activators can replace the requirement of FBS in the adipogenesis of 3T3-L1 cells. Biochem Biophys Res Commun. 2004;324: $355-9$.

21. Kawai M, Namba N, Mushiake S, Etani Y, Nishimura R, Makishima M, et al. Growth hormone stimulates adipogenesis of 3T3L1 cells through activation of the Stat5A/5B-PPARgamma pathway. J Mol Endocrinol. 2007;38:19-34.

22. Sugii S, Olson P, Sears DD, Saberi M, Atkins AR, Barish GD, et al. PPARgamma activation in adipocytes is sufficient for systemic insulin sensitization. Proc Natl Acad Sci USA. 2009;106: 22504-9. 